\title{
Characterising the Behaviour of the Ctenophore Pleurobrachia pileus in a Laboratory Aquaculture System
}

\author{
Amy Courtney ${ }^{\mathrm{a}, \mathrm{b}}$, George O.T. Merces ${ }^{\mathrm{a}, \mathrm{b}}$, and Mark Pickering ${ }^{\mathrm{a}, \mathrm{b}, 1}$ \\ ${ }^{a}$ School of Medicine, University College Dublin, Ireland. \\ bUCD Centre for Biomedical Engineering, University College Dublin, Ireland \\ ${ }^{1}$ Corresponding Author
}

\begin{abstract}
Neurobiological research focuses on a small number of model organisms, broadening the pool of animals used in research may lead to important insights into the evolution of nervous systems. The ctenophore is emerging as a promising model, but we are currently lacking an understanding into the relationship between behaviour and environment which is in part due to a lack of a standardised long-term laboratory husbandry system. We established a collection and husbandry system for wild caught Pleurobrachia pileus. We examined the behavioural profile of the animals over time in this controlled environment. We could reliably catch them on a seasonal basis, and we could keep the animals alive in our specialised aquarium system for months at a time. P. pileus spends most of the time in an inactive 'drifting' state which is interspersed with periods of one of 5 active behaviours. The most common active behaviours are tentacle resetting and feeding. The longest duration behaviours include swimming up or down. Time of day does not appear to alter their behavioural profile. Gaining a better understanding of the behaviour of these animals has important implications for systems and evolutionary neuroscience.
\end{abstract}

ctenophore | husbandry | model organism | evolution | behaviour

Correspondence: mark.pickering@ucd.ie

\section{Introduction}

Traditionally, the term "model organism" referred to an animal that was "simpler" than humans that was amenable to experimentation and facilitated investigation of a specific biological process. The meaning of the term has changed in recent years and is now often used to describe an organism which is utilised by many researchers and for which a significant experimental toolbox is available. Thus, originally model organisms were selected for suitability and convenience while now their selection is based on resource availability [Russell et al., 2017]. The catalogue of life [Roskov Y. et al., 2019] currently lists $\sim 1.3$ million animal species and it has been predicted that the true value is closer to $\sim 7.7$ million [Moraet al., 2011]. Using neuroscience as an example, 75\% of research focuses on rat, mouse and human brains with invertebrates accounting for 7.9\% [Manger et al., 2008]. This is striking when you take into consideration that invertebrates make up 95\% of all extant animal life [Lewbart, 2011]. While recent statistics on the prevalence of specific model organisms in neuroscience are not available, it appears that the animals most commonly used include human, rats, mice, fruit flies (D. melanogaster), zebrafish (D. rerio) and roundworms (C. elegans) [Borniger, 2015, Maximino et al., 2015, Chen, 2019]. While focusing research efforts on a small number of organisms has advantages, it is important to acknowledge the limitations and disadvantages that come with this approach which could be overcome by broadening the pool of model organisms used. In addition, the established model organisms may not be appropriate for answering all questions. The Krogh principle states that amongst the diversity of life there is one or multiple organisms which is most appropriate for answering a specific physiological question [Krogh, 1929]. To that end, we need to expand our repertoire of model organisms to include animals at specific positions on the phylogenetic tree while also taking into consideration the new opportunities they may present to answer specific neurobiological questions.

Members of the phylum Ctenophora are emerging as promising model organisms for comparative neurobiological investigations. Their position on the phylogenetic tree is contentious with some studies placing them as the sister taxon to all animals, while others support the sponge-first scenario [Dunn et al., 2008, Ryan et al., 2013, Feuda et al., 2014, Moroz et al., 2014, Borowiec et al., 2015, Pisani et al., 2015, Arcila et al., 2017, King and Rokas, 2017, Simion et al., 2017]. It has also been suggested that these animals may have evolved their nervous system independently to all animals [Moroz et al., 2014], however this claim remains controversial [Marlow and Arendt, 2014, Jékely et al., 2015]. Thus, these animals are either our best example of what the first nervous system looked like, or they represent the only example of divergent nervous system evolution. Gaining a better understanding of these animals will undoubtedly provide interesting insights into the foundational principles of neural structure and function. In Irish waters the most common species of ctenophore is Pleurobrachia pileus [Müller, 1776] or the sea gooseberry [Neal, 2005]. Ctenophores possess other features which allude to their promise as a model organism. They are small $(0.1 \mathrm{~mm}-20 \mathrm{~mm})$ and transparent which facilitates imaging studies, they have relatively fast 
reproduction rates [Greve, 1970, Baker and Reeve, 1974, Martindale, 1987] which facilitates laboratory rearing and genomic/transcriptomic data is available [Ryan et al., 2013, Moroz et al., 2014] which aids in the development of genetically engineered organisms. From a neurobiological perspective, they appear to possess a structurally simple and tractable nervous system, a nerve net distributed across their body [Hernandez-Nicaise, 1973a, b, Jager et al., 2011]. Despite this 'simple' neural architecture they display surprisingly sophisticated behaviours [Tamm, 2014]. However, the behaviours appear to be simple and predictable enough to examine quantitatively. Despite the apparent advantages these animals possess as a model organism, there are many unanswered questions concerning their nervous system.

The nervous system evolved to enable animals to quickly adapt to challenges they faced in their specific ecological niches. Decades of behavioural research in conventional model organisms has led to a deep understanding into the relationship between behaviour and environment. However, for unconventional model organisms, such as $P$. pileus, the relationship between behaviour and environment is less clear. For example, the sensory capabilities of these animals are unclear and in-depth analysis of their behavioural repertoire over time has never been performed. Developing a baseline behavioural profile for P. pileus will allow us to assess their amenability as a model organism and may elucidate some of the sensory mechanisms triggering these responses. To achieve this, we need a controlled environment. However, as an unestablished model organism, a standardised longterm husbandry system has never been comprehensively described. In order to promote the use of these animals as a model organism for neurobiology and to understand how their behaviours relate to their umwelt, it is imperative that we comprehensively describe the aquaculture system used to study wild caught $P$. pileus, and the characterise the behaviours displayed in this environment.

\section{Methods}

\subsection{Pleurobrachia pileus Collection}

Ctenophores were collected from the Irish Sea primarily from Howth Harbour (53⒉ $\left.34.9^{\prime} \mathrm{N} 6^{\circ} 03^{\prime} 58.0^{\prime \prime} \mathrm{W}\right)$ from September 2015 to August 2019. The highest density of animals occurred from April to December (Figure 1A) and lower densities in January and March, using a combination of collection techniques. Specifically, we used both commercial (NHBS Ltd) and custom-made plankton nets with weighted closed cod ends (1.2 - 2.2L capacity), 960-1000mm in length with $250 \mu \mathrm{m}$ and $500 \mu \mathrm{m}$ mesh size. We also used $2.5 \mathrm{~mm}$ mesh dip nets and dip cups when animals were at high enough density to be visible in the water. Animals were transported from the collection site in $2 \mathrm{~L}$ containers, at a maximum density of 150 animals/L. Time from collection to transfer to aquarium was $\sim 3$ hours, and temperature was not controlled during this time. Animals were not acclimatised to aquarium water before transfer, but we verified that the salinity in the transportation vessel and the aquarium system was comparable (30 \pm 2 ppt).

\subsection{Establishing and Maintaining the Aquaculture Sys- tem}

A detailed parts list can be found in supplementary table 1 and each component has a designator which will be referred to throughout this text. We established an aquaculture system for Pleurobrachia pileus on a bench surface in a multi-purpose laboratory environment (Figure 1B). Due to this, light dark cycle and room temperature was not tightly controlled. Animals were maintained in kreisel tanks (AC1: diameter: 400mm, depth: 200mm; Schuran Seawater Equipment, Jülich Germany). A circulating flow which maintains the animals suspended in the water column is generated by water entering through the spray bar and draining through the centrally positioned filter at the back of the tank (diameter: $15 \mathrm{~mm}$, pore size: $3 \mathrm{~mm}$ ). This white filter was covered with a $400 \mu \mathrm{m}$ black mesh which increased visibility of the largely transparent animals while also maintaining artemia nauplii in the tanks by preventing their removal through the larger filter pores. Artemia nauplii (AM12) are delivered from a holding tank (AC18) at 20-minute intervals for continuous automated feeding (using an Arduino controlled pump). Water drains from the kreisel tanks to the reservoir (AC13) initially passing through a $100 \mu \mathrm{m}$ mesh size filter sock (AC8). Live rock (AC7) and the filter start solution (AC16) provided the nitrifying bacteria. To increase the surface area upon which these bacteria can colonise filter balls (AC11) were incorporated into the reservoir. Other filtration components include a protein skimmer (AC12) and carbon (AC10) which are present in the reservoir. The water is then pumped (AC4) into a UV steriliser (AC6) and subsequently into a chiller (AC2). The chiller is set to $9 \circ \mathrm{C}$ however it fluctuates throughout the year depending on the temperature in the room. It usually stabilises around $12 \circ \mathrm{C}$ however temperatures up to $17 \circ \mathrm{C}$ were observed on multiple days during the summer months. The cooled water then recirculates back into the reservoir and is subsequently pumped (AC3) back into the kreisel tanks. Flow was maintained at approximately $600 \mathrm{ml} / \mathrm{min} / \mathrm{tank}$. Flow to each kreisel can be independently controlled or stopped for cleaning or decommissioning. Each kreisel has a $\sim 28 \mathrm{~L}$ capacity and the capacity of the entire system is usually $\sim 150 \mathrm{~L}$. We use fresh seawater (Seahorse Aquariums Ltd.) and a 25L water change is performed every 3 weeks. Cleaning procedures are performed monthly and involves scrubbing the inner surface of the kreisel tanks and spray bar to remove any debris which has accumulated. No chemicals are used during cleaning.

Animals were added to the aquaculture system and removed for experimentation at various time points, allowing us to calculate the expected number of animals in the system at any point in time. The actual number of animals was measured and showed a decline over time (Figure 1C). This suggests that while the aquaculture system may not be optimal for long term survival of $P$. pileus in the laboratory, it is possible to maintain animals for extended periods of time (i.e. several months), which is sufficient for the experimental aims of this study. 
A

\section{P. Pileus Collection Rates in Howth Harbour}

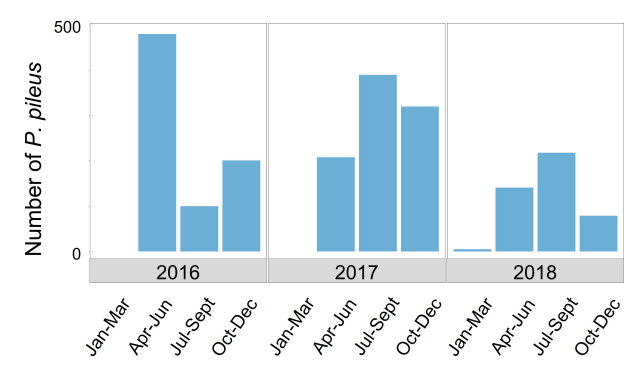

C

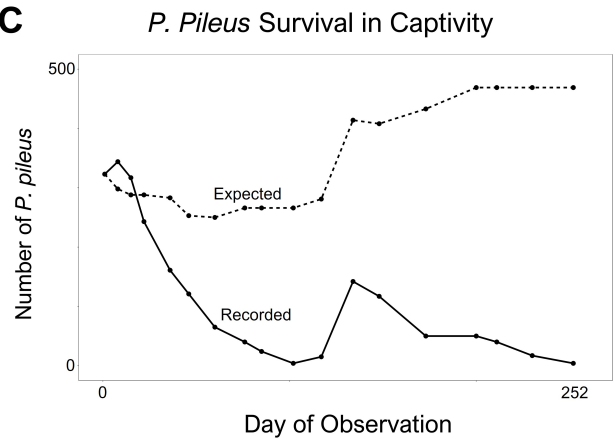

B

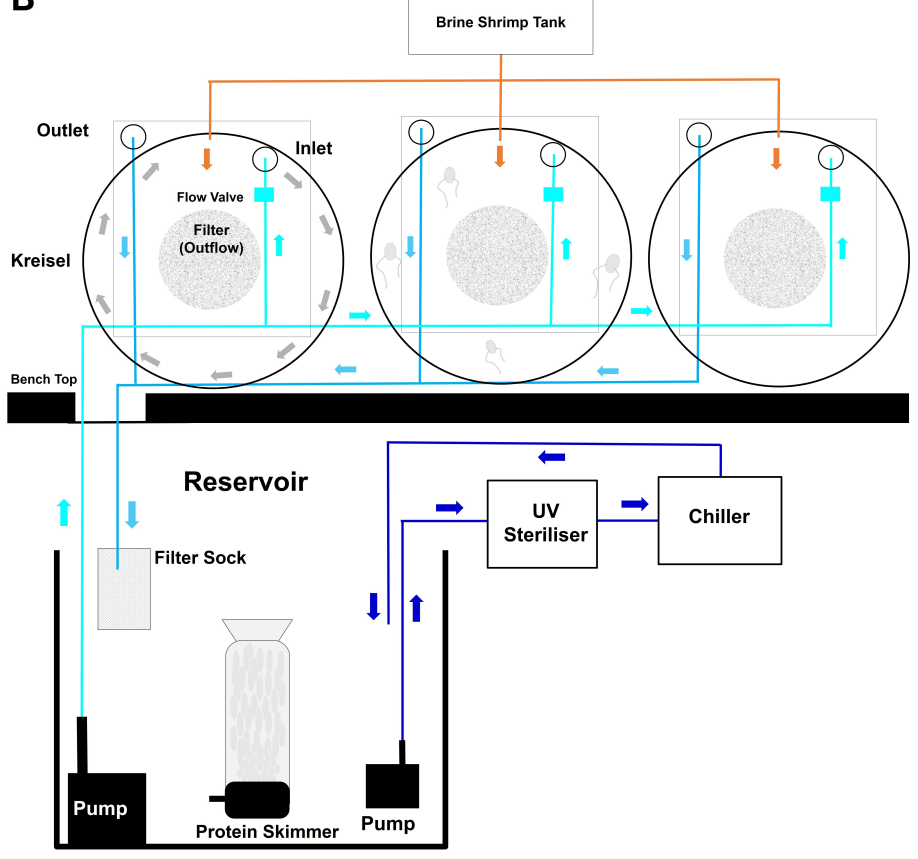

Figure 1. Pleurobrachia pileus Collection and Husbandry. (A) Over the course of three years collecting $P$. pileus at Howth harbour we observed a seasonal occurrence of animals, highest densities from April-December and little to no animals in January-March. (B) This is a schematic representation of the husbandry system we established for wild caught $P$. pileus. A parts list for all components in this schematic can be found in Supplementary Table 1 . Animals were maintained in kreisel tanks which have a circulating flow which maintains the animals suspended in the water column. Water flows in through the spray bar and is drained through the filter at the back of each tank. An Arduino controlled pump system delivers Artemia nauplii from a holding tank at 20-minute intervals. Water drains from the kreisel tanks to the reservoir initially passing through a filter sock. Live rock, filter balls, a protein skimmer and carbon are present in the reservoir and provide filtration. The water is then pumped into a UV steriliser and subsequently into a chiller (set to $9 \circ \mathrm{C}$ ). The cooled water then recirculates back into the reservoir and is subsequently pumped back into the kreisel tanks. Each kreisel possesses a valve to adjust flow. The system has a $150 \mathrm{~L}$ capacity and we use fresh seawater. (C) The recorded number of animals present in our system compared to the expected number. The expected values relate to the predicted number of animals present assuming no death while also taking into account the number of animals removed for experiments and added after subsequent collection trips. The discrepancy between the recorded and expected values means that the number of animals is decreasing over time due to death.

\subsection{Behavioural Monitoring}

Animals used for behavioural experiments were caught in October 2018 and had been in the tanks for 18 days before the experiment began. The animals were subjected to a 12-hour light dark cycle for the duration of the experiment. 13 animals were present in one kreisel tank (one animal per $1.9 \mathrm{~L}$ ), and 10 minute videos were acquired of this single tank at the start $(7 \mathrm{am})$, mid $(1 \mathrm{pm} \pm 1$ hour) and end $(7 \mathrm{pm}$ \pm 1 hour) of the light cycle over a four-day period. No data was collected during the dark cycle. The same animal could not be tracked from one video to another. Specific, discrete behavioural states were defined: the active behaviours observed in this environment were tentacle resetting, feeding, swimming up, swimming down, and escape swimming, while drifting was considered their default 'inactive' behavioural state (supplementary table 2 , supplementary video 1). Videos were manually annotated with Kinovea software [Charmant] with the time and location of transition between behavioural states in each animal. Periods of lost data or unclassifiable behaviours due to environmental interference and motionlessness were also noted. Motionlessness refers to animals coming into close contact with the black mesh on the filter outflow and staying in a fixed position for periods of time. Environmental interference relates to animals with a tentacle attached to the inner surface of the tank or to another animal.

\subsection{Statistical Analysis and Data Visualisation}

The statistical analysis and data manipulation was performed using R [Core Team, 2018]. R packages utilised for data visualisation include ggplot2 [Wickham, 2016], ggthemes [Arnold, 2019], cowplot [Wilke, 2019] and networkD3 [Allaire et al., 2017]. For behavioural analysis, we examined the frequency and duration of individual behaviours, the temporal sequence of behaviours, and the location within the tank in which specific behaviours occurred.

\section{Results}

\subsection{Behaviour Distributions Across the Light Cycle}

The sequence of behaviours of each of the 13 animals over each of the 10 minute recordings at the start, middle and end of the light cycle over 4 days is shown in Figure 2A. Pooled across all recordings, the majority of the time is spent drifting (inactive behaviour); $74 \%$ of the total time analysed in the dataset was spent drifting, $\sim 8 \%$ was unclassifiable and $\sim 18 \%$ was spent actively behaving. Of that, tentacle resetting accounted for $\sim 9.2 \%$, feeding was $\sim 2.4 \%$, swimming down 
equated to $\sim 6 \%$, swimming up was $\sim 0.5 \%$ while escape swimming was $\sim 0.2 \%$. When this is segregated into the start, middle and end of the light cycle, there is an apparent decrease in the total amount of time spend engaged in active behaviours as the light cycle progresses (Figure 2B). This could result from changes in the duration of behaviours, or changes in the number of times a behaviour is initiated. However, when the total number of behavioural initiations is pooled, there is an apparent increase in initiations as the light cycle progresses (Figure 2C). Therefore, a more complete understanding of the behavioural distribution requires that both the duration and frequency of individual behaviours are examined separately.

\subsection{Active Behaviour Duration}

We examined whether the duration of a behaviour varied across the light cycle by comparing the durations of all instances of that behaviour across the three time points recorded (Figure 2D). We found no difference between the duration of behaviours at different points in the light cycle for either of the two most common behaviours, namely tentacle resetting (Kruskal-Wallis rank sum test $(\mathrm{KW})$; $2=353.01, \mathrm{p}=0.17, \mathrm{df}=329, \mathrm{n}=671)$ and feeding behaviour $(\mathrm{KW} ; 2=140.50, \mathrm{p}=0.31, \mathrm{df}=133, \mathrm{n}=168)$.

Similarly, we found no difference in swimming down across the light cycle (KW; $2=19, \mathrm{p}=0.46, \mathrm{df}=19, \mathrm{n}=20$ ). However, it should be noted that only two instances of this behaviour were recorded at the end of the light cycle. Swimming up was also a relatively rare behaviour with no instances observed at the start of the light cycle position, but no difference in duration was found between the middle and end of the light cycle $(\mathrm{KW} ; 2=5, \mathrm{p}=0.42, \mathrm{df}=5, \mathrm{n}=6)$. Another rare behaviour was the escape swimming behaviour which had only one instance at the end of the light cycle. Again, no difference was observed between the three time points (KW; $2=11, \mathrm{p}=0.44, \mathrm{df}=11, \mathrm{n}=12$ ). We also tested if drifting duration varied at different times of the day and found no difference $(\mathrm{KW} ; 2=680.08, \mathrm{p}=0.39, \mathrm{df}=670, \mathrm{n}=753)$.

\subsection{Active Behaviour Count}

While we found no evidence that the duration of instances of active behaviours changed across the light cycle, we also examined whether the frequency of initiation of these behaviours changed over the light cycle. This was assessed by counting the number of times a behaviour was initiated by each animal in a ten-minute recording (Figure 2E). While there was no change in tentacle resetting count across the light cycle $(\mathrm{KW} ; 2=13.73, \mathrm{p}=0.19, \mathrm{df}=10, \mathrm{n}=156)$ the count of feeding behaviours did show changes, with feeding behaviour initiated more often at the end of the light cycle $(\mathrm{KW} ; 2=15.86, \mathrm{p}=0.01, \mathrm{df}=6, \mathrm{n}=156)$.

The other three behaviours (swimming up, swimming down, and escape swimming) were, as noted earlier, rarer events. The most common outcome was animals where no incidences of these behaviours were recorded in the 10 minute period of observation. Nonetheless, we noted a difference in the frequency of swimming down behaviours, with higher counts seen at the start of the light cycle (KW; $2=12.72, p=0.01, d f=4, n=156$ ), while the opposite was true of swimming up behaviours, which had higher counts at the end of the light cycle (KW; $2=8.06, p=0.02, \mathrm{df}=2, \mathrm{n}=156$ ), and no instances of swimming up observed at the start of the light cycle. On the other hand, no differences were found between the counts of escape swimming at each light cycle position $(\mathrm{KW} ; 2=3.14, \mathrm{p}=0.30, \mathrm{df}=2, \mathrm{n}=156$ ). When we examined the behaviour count of drifting at different light cycle positions, we also found no difference (KW; $2=13.83$, $\mathrm{p}=0.31, \mathrm{df}=12, \mathrm{n}=156$ ).

\subsection{Examining the Sequential Relationships Between Behaviours}

In addition to the frequency and duration of individual behaviours, we also explored how individual behaviours might sequentially influence each other. Specifically, we examined whether individual behaviours were linked into sequential repertoires, which could be identified by repeating sequences of individual behaviours. To do this, we looked at each instance of an active behaviour initiation after a period of drifting (first behaviour), and examined the next behaviour initiated (second behaviour). Two behavioural sequences accounted for the majority of all observations at the start, middle and end of the light cycle: tentacle resetting followed by drifting (Start: 76\%, Mid: 78\%, End: 78\%) and feeding followed by drifting (Start: $21 \%$, Mid: 17\%, End: 19\%) (Figure 3A). This indicates that active behaviours are not usually chained into sequences, but rather are initiated after a period of drifting, and end with the animal returning to an inactive drifting state before another active behaviour is initiated.

While one behaviour did not typically lead directly to the initiation of another behaviour, it remained possible that the initiation of one behaviour could still influence the next active behaviour to be initiated after a period of drifting; specifically, we wanted to determine if the occurrence of either of the two most common active behaviours (feeding or tentacle resetting) predicts the next behaviour initiated after a period of drifting. For example, are animals more like to initiate a tentacle reset after a period of feeding?

Pooled across all observations, resetting behaviours (780 in total) were initiated more commonly than feeding behaviours, (149 in total), giving a percentage ratio for these behaviours of $84 \%: 16 \%$. When feeding was initiated, the next behaviour initiated was also feeding in $17.5 \%$ of cases, while it was resetting in $82.5 \%$ of cases. When resetting was initiated, the next behaviour initiated was resetting in $81.5 \%$ of cases, while it was feeding in $18.5 \%$ of cases (Figure 3B). There is no evidence that either behaviour predicts the sequential occurrence of the other (Pearson's Chi-squared test; $2=1.39, \mathrm{df}=2, \mathrm{p}=0.50$ ), leaving us to conclude that the behaviours are independent of each other.

\subsection{What Triggers a Tentacle Resetting Event?}

The trigger for the feeding behaviour is well understood: prey captured on a tentacle initiates a cascade of events 
A

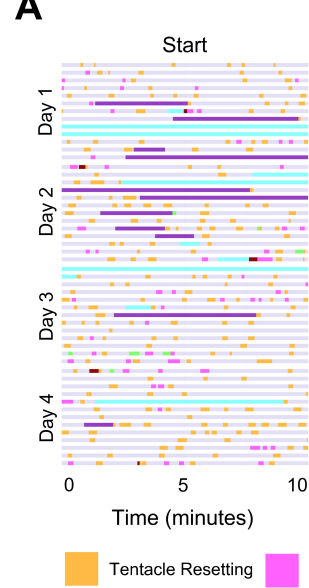

Light Cycle Position

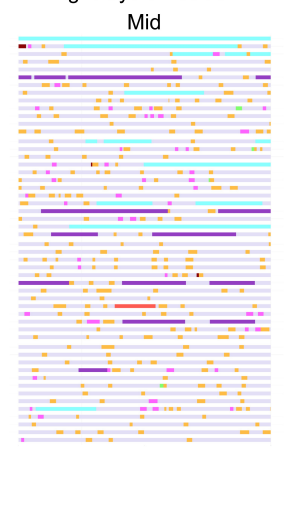

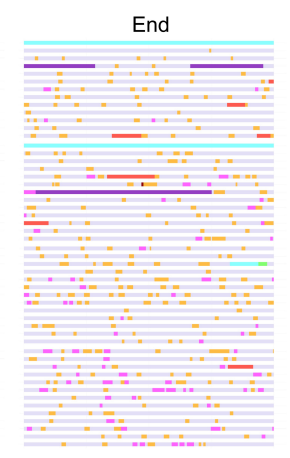

B

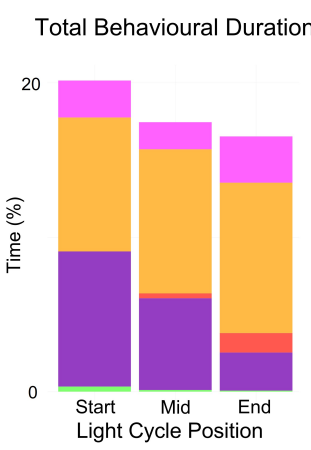

C

Total Behavioural Count

D

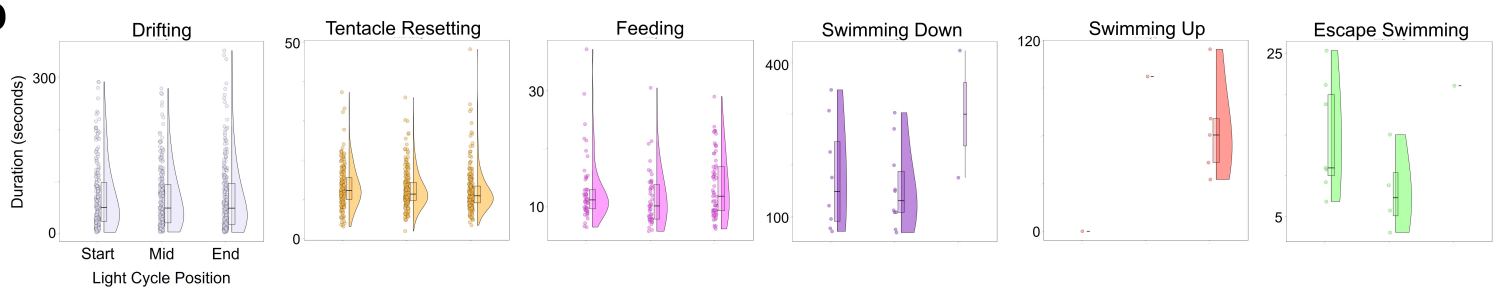

\section{E}
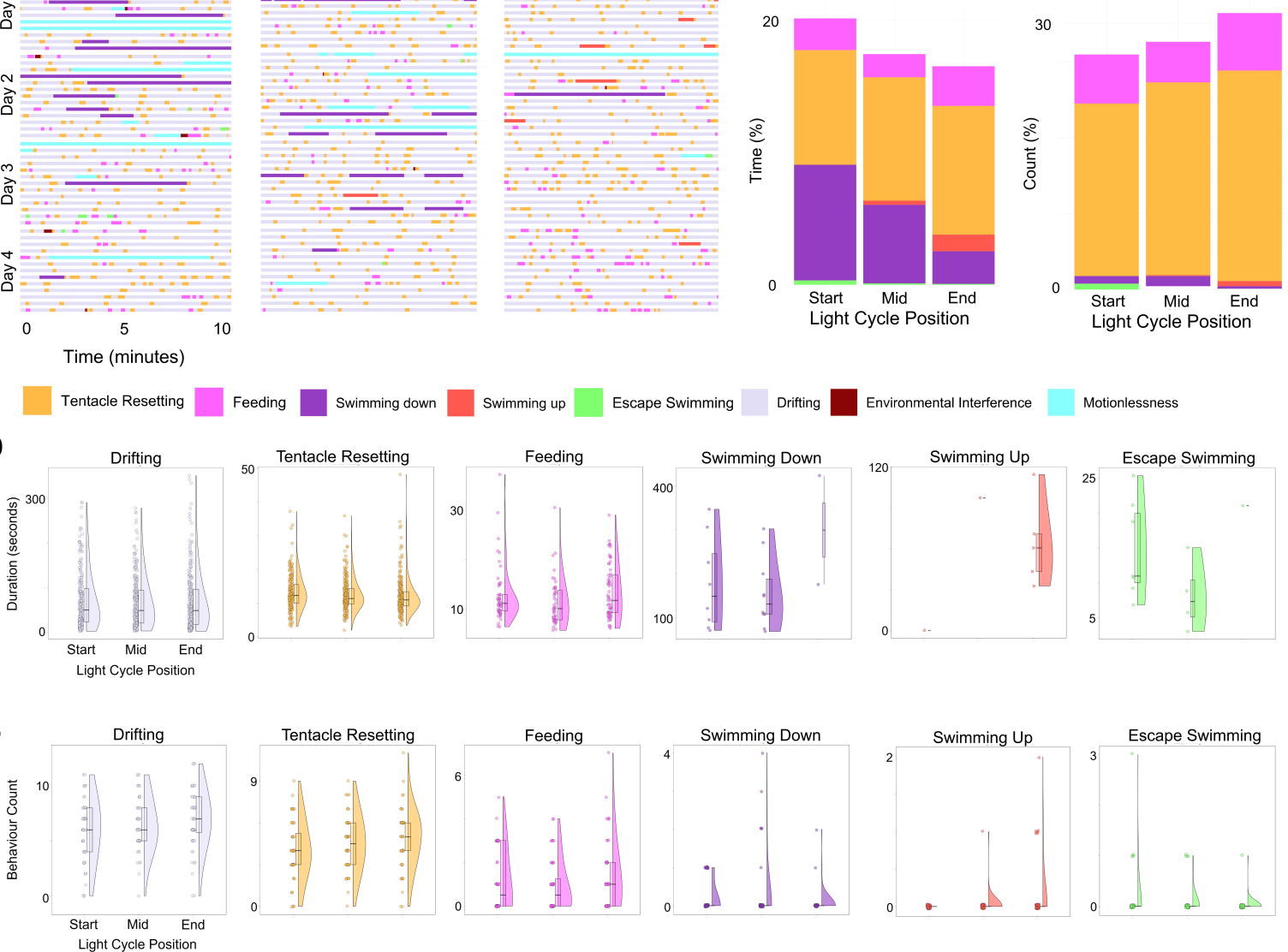

Motionlessness 


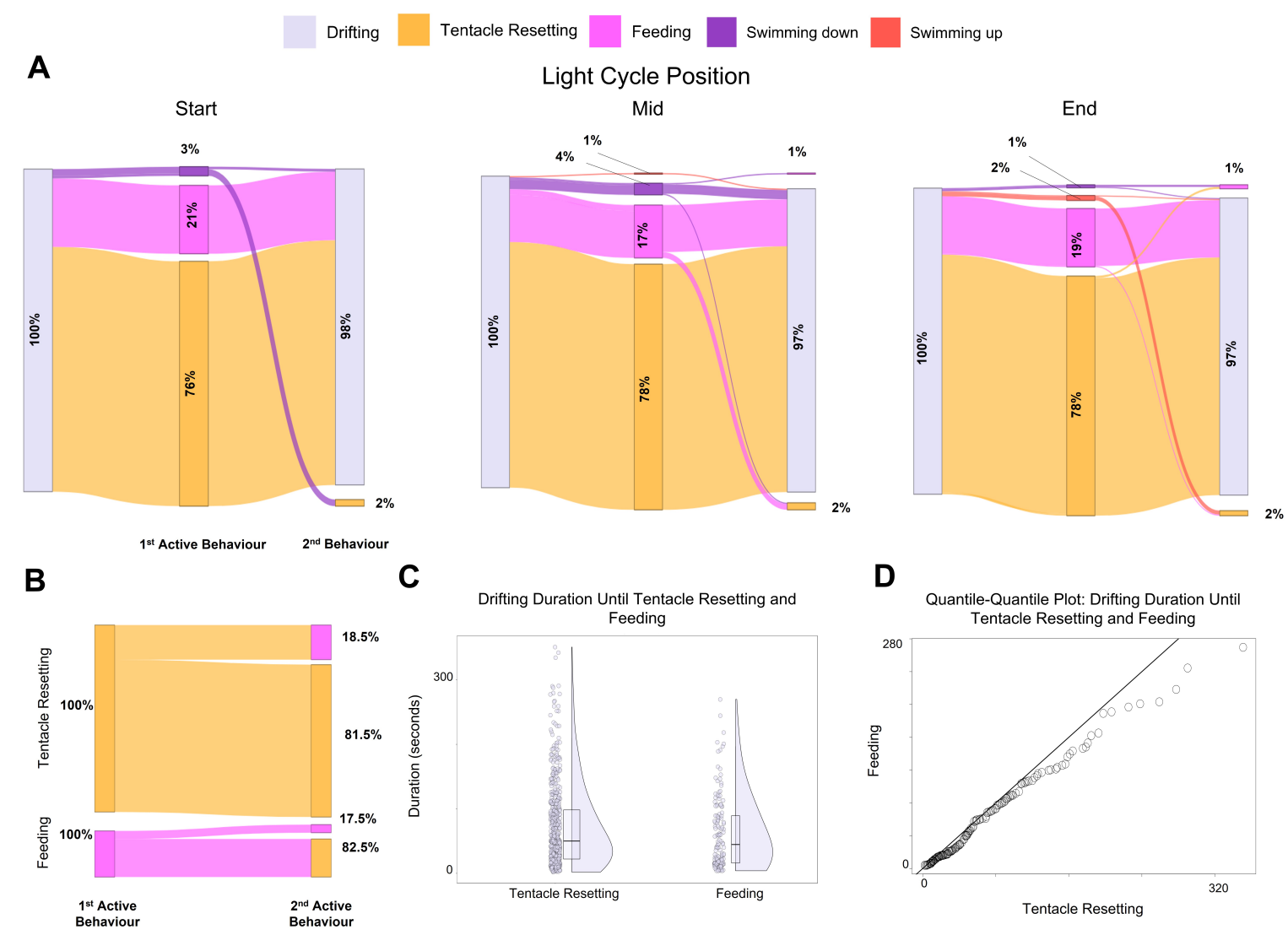

Figure 3. Does a preceding behaviour predict a proceeding behaviour? (A) We extracted all instances of an active behaviour after drifting (1st behaviour) and showed the following behaviour which proceeded it (2nd behaviour). The events are displayed as a percent of all instances in each light cycle position. We next wanted to examine what drives the most common sequence of behaviours and whether one behaviour could predict another. (B) The sequential characteristics of behaviours which begin with a tentacle resetting or feeding behaviour. The events are displayed as a percent of all instances of a sequence beginning with either tentacle resetting or feeding. (C) We pooled all instances of drifting duration until tentacle resetting and feeding across all light cycle positions. This plot includes the individual data points, a half violin plot to visualise the distribution and a boxplot which includes the median value as the middle horizontal line and the 25th and 75th quartile range as the lower and upper horizontal lines respectively. (D) A quantile-quantile plot compares the distributions of drifting interval until tentacle resetting and drifting interval until feeding. No difference between the groups was observed.

horizontal flow at the top of the tank, region 2 involves downward flow on the right of the tank, region 3 includes horizontal flow at the bottom of the tank, region 4 includes upward flow on the left of the tank and region 5 is in the centre of the tank directly in front of the outflow filter. The inflow spray bar was located in region 1 close to the border with region 2. Given the direction of circulation, we assume therefore that region 2 has the most turbulent flow.

We noted the location where each of the feeding and tentacle resetting events were initiated. By comparing the distribution of behavioural initiations across the 5 regions to the distribution of time the animals spent in each region, we could determine if behavioural initiations were more likely to occur in specific regions. While each region was of the same volume, the time animals spent in each region (the "expected" value for each region) was not equal. The "expected" value was generated by manually counting the distribution of animals every minute in all videos (13 animals at each of the 120 observations) irrespective of behaviour (Figure 4A). We found a significant difference between the distribution of resetting events and overall distribution of the animals (Pearson's Chi-squared test; $2=38.94, \mathrm{df}=4, \mathrm{p}<0.0001)$. It appears that there is a higher probability that animals will initiate tentacle resetting in region 2 (Figure 4B/C). However, we found no difference between the regional distribution of feeding events and the overall distribution of the animals (Pearson's Chi-squared test; $2=5.52, \mathrm{df}=4, \mathrm{p}=0.24$ ). It appears the probability of a feeding event occurring in any location in the tank is equal (Figure 4D/E).

\section{Discussion}

We have comprehensively examined the Pleurobrachia pileus behavioural repertoire. We analysed the relationships between behaviours, behavioural durations, behavioural frequencies and spatial characteristics of behaviours. This was done to elucidate possible intrinsic and extrinsic mechanisms which drive these actions and also to examine the affect time 

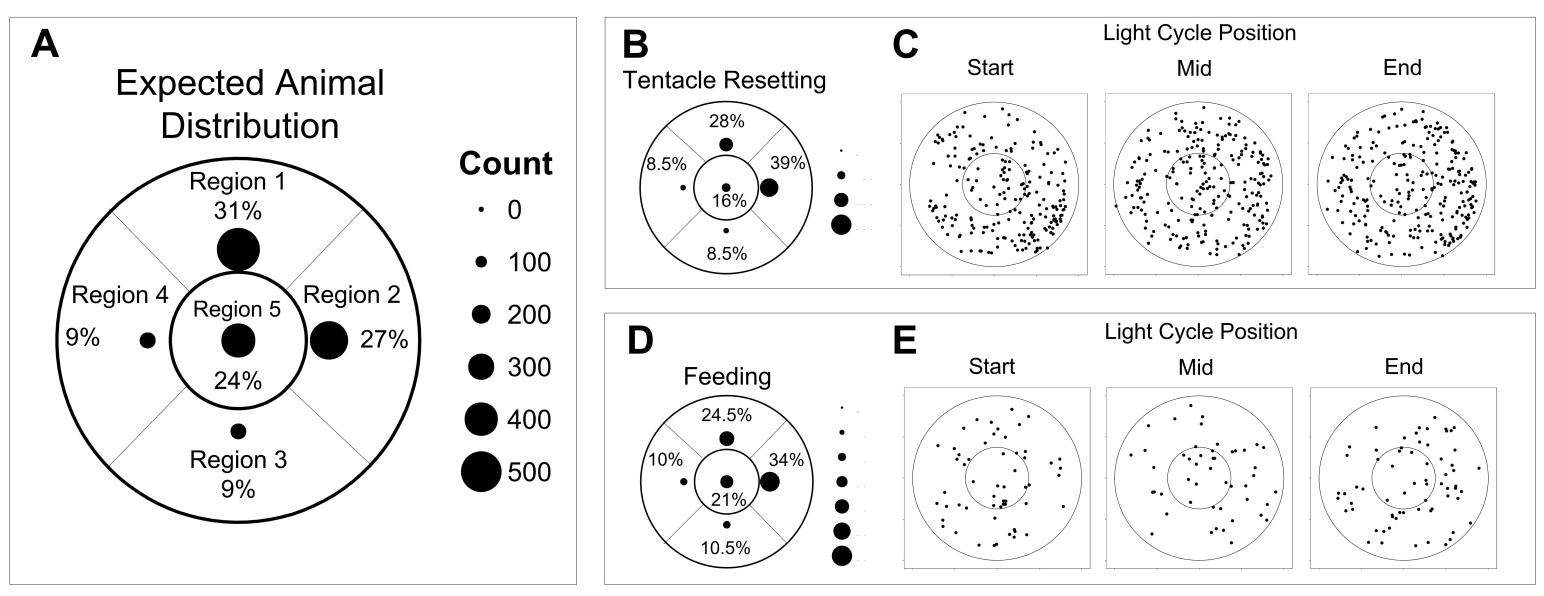

Figure 4. Spatial Characteristics of Behaviours. Does the animal's location in the tank predict the initiation of a behaviour? We divided the tank into 5 regions, largely based on the assumed fluid dynamics within the tank. We could not assume the animal distribution to be equal across all regions. (A) We calculated the 'expected' animal distribution per region by manually counting the location of animals every minute in all videos irrespective of behaviour. (B) The number and proportion of animals which initiated a tentacle resetting behaviour in each region. (C) The location within the kreisel tank upon which tentacle resetting was initiated at each light cycle position. (D) The number and proportion of animals which initiated a feeding behaviour in each region. (E) The location within the kreisel tank upon which feeding was initiated at each light cycle position. A, B D all have the same plotting conventions; the number of instances of an initiation or completion events are displayed as count (point size) and as percent of all instances across all light cycle positions. In all plots the outer circle denotes the outer edge of the tank while the inner circle denotes the edge of the centrally located outflow filter.

of day has on them. Of the five active behaviours identified, tentacle resetting and feeding were the most commonly initiated. Swimming down and swimming up were rare but had the longest durations. Behaviour duration does not vary at different times of the day but behaviour count does, for some behaviours. Most of the time was spent in the inactive drifting state. The duration of these periods of inactivity are variable and most often results in transitioning into tentacle resetting or feeding and back to drifting again. The spatial location of an animal in the tank appears to influence the initiation of tentacle resetting.

The most common active behaviour was tentacle resetting. The tentacle resetting behaviour was observed 671 times and accounted for $\sim 9.2 \%$ of the total data set. Our data suggests that tentacle resetting is not influenced by a preceding feeding behaviour, it is random in its likelihood of initiation in terms of time but not in terms of its spatial location. This mechanism of preparing the tentacle for prey capture has not been investigated in a detailed manner but has been observed before [Tamm and Moss, 1985]. The aim of this behaviour is presumably to prepare the tentacle for prey capture and performing these motions while relaxing the tentacles and tentilla appear to provide a large surface area. The animals were more likely to initiate a tentacle resetting bout in tank region 2 , which due to being downstream of the water inlet is likely to have the most turbulent flow. It seems most likely that this behaviour is not internally generated, but rather is a response to external stimuli, but the nature of the external stimuli are not clear. However, it seems likely that water flow and turbulence, which may impact on the tentacles, is a significant factor in initiating this behaviour.

Feeding and tentacle resetting are closely associated behaviours as they both contribute to successful ingestion of prey. The feeding behaviour in Pleurobrachia has been de- scribed comprehensively in both free swimming [Tamm and Moss, 1985] and restrained animals [Moss, 1991, Moss and Tamm, 1993]. Pleurobrachia feeding behaviour was observed 168 times and accounted for $\sim 2.4 \%$ of our total data set. The overall frequency observed in our analysis may be due to many factors; the density of artemia, the kreisel volume and the density and size of $P$. pileus during the time of data collection [Gibbons and Painting, 1992]. It is widely accepted that Pleurobrachia is a voracious non-selective predator. Feeding behaviour duration did not vary with the time of day but it did vary in terms of frequency at different time points. This difference may be due to the density of artemia in the tanks. The location of Pleurobrachia in the tank does not predict the initiation of a feeding behaviour, which is unsurprising if the prey are distributed in the tanks in a similar manner to the animals. In addition, our results which indicate that a previous tentacle resetting or feeding event did not influence a subsequent feeding event demonstrates the voracious predatory nature of the animals. They do not appear to be using their previous ingestion of prey to prevent a subsequent ingestion. Perhaps at higher food availability their satiety would impact the initiation of a feeding behaviour. However, we have witnessed animals purging gut material and this may be the only mechanism they have to control their food intake. While tentacle resetting and feeding were the dominant active behaviours, we also observed other distinct behaviours that, while rarer, may also play important roles in ctenophore biology, although the actual function of the behaviours is less clear. Swimming up and down are two behaviours which fall into this category. Animals which demonstrated swimming up and swimming down behaviours had to be undertaking this act for more than 28 seconds to be constituted as such because feeding and tentacle resetting were also associated with preceding directional swimming. These behaviours are 
the longest duration active behaviours, but they are not very common. Six instances of swimming up and twenty instances of swimming down were noted from the entire data set. In all cases of swimming up, it exclusively occurred on the right side of the tank as the animals swam against the water flow. In contrast, swimming down exclusively occurred on the left side of the tank where they again were swimming against the water current. Swimming up equates to $\sim 0.5 \%$ of the total data set while swimming down equates to $\sim 6 \%$. However, we must consider the impact the tank walls will have on these behaviours. Perhaps the durations and counts would be higher in the wild with less obstacles disturbing the response. Encountering the tank walls during this behaviour may cause shorter durations. The aquaculture system we describe here may not be ideal for studying these specific behaviours. We observed that the duration of the swimming up and down events did not vary with the time of day but the behaviour counts did. While it is tempting to link this observation to possible diurnal migration, we must be cautious in drawing conclusions from relatively rare behaviours.

The behaviours which we referred to as escape swimming we believed encompassed high frequency active swimming behaviours that did not fall into the realm of the other active behaviours and that were more 'erratic' in nature. They are relatively rare (12 instances and $\sim 0.2 \%$ of total data set). There was no difference in duration or count of this behaviour at any of our sampled time points. However, we did observe that $83.5 \%$ of initiation events occurred in region 5. Region 5 encompasses the filter outflow; perhaps body wall or tentacle interactions with a filter pore may have driven this behaviour. Our classification of an 'escape' behaviour appears to closely resemble behaviours in the literature. Global ciliary excitation is described as a fast forward swimming response [Tamm, 1982]. Global ciliary excitation can also occur in reverse [Tamm and Tamm, 1981, Mackie et al., 1992, Tamm and Terasaki, 1994]. Our results indicated that there isn't a substantial difference in behavioural characteristics at different times of the day. Our analyses may have missed specific features of a circadian rhythm, or the aquarium environment may have altered their natural circadian rhythms, so we cannot rule out the existence of a circadian rhythm in these animals. Circadian rhythms are influenced by light, but direct physiological evidence of light-sensing cells in Pleurobrachia does not exist. Chun [1880], Horridge [1964] and Hernandez-Nicaise [1984] described structures resembling photoreceptors in the aboral organ. In addition, a more recent investigation in Mnemiopsis leidyi found opsin expression co-localised with these structures which supports their role as photoreceptors [Schnitzler et al., 2012]. Ctenophores have been shown to respond to light behaviourally; spawning [Freeman and Reynolds, 1973, Pianka, 1974, Pang and Martindale, 2008, Patry L. et al., 2019] and reversing geotaxis [Tamm, 1982].

This list of behaviours in our analysis is not exhaustive, due to the nature of our data collection and analyses there were certain behaviours we could not identify and may have been included under the umbrella of others. For ex- ample, mechanical stimulation to any location on Pleurobrachia's body results in temporary interruption of beating of all the comb plates, this has been referred to as sudden stoppage/global ciliary inhibition/primary inhibition [Moss, 2004]. Another response not noted in our data set is secondary inhibition, stronger stimulation (as compared to primary inhibition) leads to muscular contraction of the comb rows inward presumably to protect the important locomotory structures [Tamm and Tamm, 1989]. Subtler actions such as body wall muscular contractions, mouth movements, defaecation [Presnell et al., 2016] and gamete release [Pianka, 1974, Patry L. et al., 2019] could also not be captured in this analysis.

Our results also show that collecting a sufficient number of animals is feasible and reliable at Howth Harbour, but their density is seasonal. Changes in overall density of Pleurobrachia at different times of the year and between years has been observed by many researchers [Hirota, 1974, Yip, 1984, Frank, 1986, Buecher and Gasser, 1998]. The seasonal peaks of P. pileus in different locations was summarised by Wang et al. [1995] with the highest densities for most reported in May and June. This seasonality makes a long-term aquaculture system necessary for reliable, year-round experimentation. Our aim was not to develop an optimal aquaculture system, but to develop one that was simple, robust and sufficient for our purposes, specifically providing long term reliable access to animals without necessitating on demand collection at sea, and allowing observation of animals for a period of several weeks at least. The survivability data is challenging to interpret as the life span of P. pileus in the wild is unknown. Factors which may have impacted the longterm survival and behaviour of these animals includes animal density, food source, temperature, lighting, light-dark cycle and water quality. Potential improvements include rigorous monitoring of environmental variables and assessing the impact of animal density and food sources on survival and behaviour. Future studies could take advantage of a deep neural network approach to tracking animal trajectory [Mathiset al., 2018] and the development of a machine learning algorithm could also enable an unbiased method of categorising behaviours [Han et al., 2018]. These tools would enable longterm and high-throughput behavioural analysis. A successful ctenophore culture protocol has recently been established at the Monterey Bay Aquarium [Patry L. et al., 2019] which could easily be combined with the system we described here. However, many benefits exist to studying wild caught animals - it indicates that their nervous system was working optimally to enable them to develop, avoid predators and acquire food.

\section{Conclusion}

We have shown that $P$. pileus can be collected reliably and in high densities at specific times of the year in the Irish Sea and that we can keep them alive in a specialised aquarium system for months at a time. We hope the description of our ctenophore husbandry system will encourage others to publish detailed accounts of their efforts in this regard. 
The optimisation and standardisation of these systems are imperative to the establishment and longevity of a successful ctenophore research community. We also characterised the full behavioural repertoire of $P$. pileus in our aquarium system. This analysis may be of interest to neuroscientists who are interested in answering questions about the foundational principles of nervous systems in a tractable model. It is unclear if our behavioural profile is comparable to their behaviour in the wild and an important next step will be to acquire a similar dataset from animals in their natural environment.

\section{Acknowledgements}

The authors wish to acknowledge Dominic Courtney for his invaluable assistance in the collection of ctenophores. The authors would also like to thank the staff at Seahorse Aquariums for their expert advice in setting up the aquaculture system.

\section{Statement of Ethics}

As they are invertebrate species, use of ctenophores in research is exempt from full ethical review under local policies and legislation. Formal exemption from review was granted by UCD AREC (AREC-E-17-22-Pickering).

\section{Disclosure Statement}

The authors declare no conflict of interest.

\section{Funding Sources}

This work was supported by School of Medicine, University College Dublin.

\section{Author Contributions}

AC collected the ctenophores, designed and built the husbandry system, carried out and analysed the behavioural and survival experiments and wrote the manuscript. GM assisted in the collection and maintenance of ctenophores and designed the automated brine shrimp feeding system. MP conceived the project, supervised all aspects of the work and edited the manuscript.

\section{Bibliography}

1. Allaire JJ, Gandrud C, Russell K, Yetman C. networkD3: D3 JavaScript Network Graphs from R. 2017.

2. Arcila D, Ortí G, Vari R, Armbruster JW, Stiassny MLJ, Ko KD, et al. Genome-wide interrogation advances resolution of recalcitrant groups in the tree of life. Nature Ecology Amp; Evolution. 2017 01/13/online;1:0020.

3. Arnold JB. ggthemes: Extra Themes, Scales and Geoms for 'ggplot2' 2019.
4. Baker LD, Reeve MR. Laboratory culture of the lobate ctenophore Mnemiopsis mccradyi with notes on feeding and fecundity. Marine Biology. 1974 1974/07/01;26(1):57-62.

5. Borniger J. Rejuvenating the Comparative Approach in Comparative Neuroscience. https://physorg/news/2015-07rejuvenating-approach-modern-neurosciencehtml2015.

6. Borowiec ML, Lee EK, Chiu JC, Plachetzki DC. Extracting phylogenetic signal and accounting for bias in whole-genome data sets supports the Ctenophora as sister to remaining Metazoa. BMC Genomics. 2015 2015/11/23;16(1):987.

7. Buecher E, Gasser B. Estimation of predatory impact of Pleurobrachia rhodopis (cydippid ctenophore) in the northwestern Mediterranean Sea: in situ observations and laboratory experiments. Journal of Plankton Research. 1998;20(4):631-51.

8. Charmant, Kinovea. Version:0.8.15. Available at: www.kinovea.org

9. Chen A. Animal Model Diversity in Neuroscience. https://wwwharvardneuroblogcom/blog/2019/3/12/animalmodel-diversity-in-neuroscience2019.

10. Chun. Die Ctenophoren des Golfo von Neapel und der angrenzenden Meeres-Abschnitte. Flora und Fauna des Golfes von Neapel. 1880; 1:[pp. 1-311. pp.].

11. Core Team R. R: A language and environment for statistical computing. Vienna, Austria R Foundation for Statistical Computing 2018.

12. Dunn CW, Hejnol A, Matus DQ, Pang K, Browne WE, Smith SA, et al. Broad phylogenomic sampling improves resolution of the animal tree of life. Nature. 2008;452(7188):745-49.

13. Feuda R, Rota-Stabelli O, Oakley TH, Pisani D. The comb jelly opsins and the origins of animal phototransduction. Genome Biol Evol. 2014 2014//;6.

14. Frank KT. Ecological Significance of the Ctenophore Pleurobrachia pileus off Southwestern Nova Scotia. Canadian Journal of Fisheries and Aquatic Sciences. 1986 1986/01/01;43(1):211-22.

15. Freeman G, Reynolds GT. The development of bioluminescence in the ctenophore Mnemiopsis leidyi. Developmental Biology. 1973 1973/03/01/;31(1):61-100.

16. Gibbons MJ, Painting SJ. The effects and implications of container volume on clearance rates of the ambush entangling predator Pleurobrachia pileus (Ctenophora: Tentaculata). Journal of Experimental Marine Biology and 
Ecology. 1992 1992/11/16/;163(2):199-208.

17. Greve W. Cultivation experiments on North Sea ctenophores. Helgoliinder wiss Meeresunters. 1970;20:30417.

18. Han S, Taralova E, Dupre C, Yuste R. Comprehensive machine learning analysis of Hydra behavior reveals a stable basal behavioral repertoire. eLife. 2018 2018/03/28;7:e32605.

19. Hernandez-Nicaise M-L. Ctenophora. In: Bereiter-Hahn J, Matoltsy AG, Richards KS, editors. Biology of the Integument: Invertebrates. Berlin, Heidelberg: Springer Berlin Heidelberg; 1984. p. 96-111.

20. Hernandez-Nicaise ML. [The nervous system of ctenophores. I. Structure and ultrastructure of the epithelial nerve-nets]. Z Zellforsch Mikrosk Anat. 1973a Feb 12;137(2):223-50.

21. Hernandez-Nicaise ML. [The nervous system of ctenophores. II. The nervous elements of the mesoglea of beroids and cydippids (author's transl)]. Zeitschrift fur Zellforschung und mikroskopische Anatomie (Vienna, Austria : 1948). 1973b 1973;143(1):117-33.

22. Hirota J. Quantitative natural history of Pleurobrachia bachei in La Jolla Bight. Fishery Bull, Wash. 1974;72(2):295-335.

23. Horridge GA. Presumed photoreceptive cilia in a ctenophore. Quart J Microsc Sci. 1964 1964//;105.

24. Jager M, Chiori R, Aliá A, Dayraud C, Quáinnec E, Manuel M. New insights on ctenophore neural anatomy: immunofluorescence study in Pleurobrachia pileus (Müller, 1776). J Exp Zool (Mol Dev Evol). 2011 2011//;316B.

25. Jékely G, Paps J, Nielsen C. The phylogenetic position of ctenophores and the origin(s) of nervous systems. EvoDevo. 2015 2015//;6(1):1-9.

26. King N, Rokas A. Embracing Uncertainty in Reconstructing Early Animal Evolution. Current Biology. 2017;27(19):R1081-R88.

27. Krogh A. The progress of physiology. American Journal of Physiology. 1929 (90): 243-51.

28. Lewbart GA. Invertebrate Medicine. John Wiley Sons, Inc.; 2011.

29. Mackie GO, Mills CE, Singla CL. Giant axons and escape swimming in Euplokamis dunlapae (Ctenophora: Cydippidea). Biol Bull Woods Hole. 1992 1992//;182.
30. Manger PR, Cort J, Ebrahim N, Goodman A, Henning J, Karolia M, et al. Is 21st century neuroscience too focussed on the rat/mouse model of brain function and dysfunction? Frontiers in neuroanatomy. 2008;2:5-5.

31. Marlow H, Arendt D. Evolution: Ctenophore genomes and the origin of neurons. Curr Biol. 2014 2014//;24.

32. Martindale MQ. Larval reproduction in the ctenophore Mnemiopsis mccradyi (order Lobata). Marine Biology. 1987 1987/04/01;94(3):409-14.

33. Mathis A, Mamidanna P, Cury KM, Abe T, Murthy VN, Mathis MW, et al. DeepLabCut: markerless pose estimation of user-defined body parts with deep learning. Nature Neuroscience. 2018 2018/09/01;21(9):1281-89.

34. Maximino C, Silva RXdC, da Silva SdNS, Rodrigues LdSDS, Barbosa H, de Carvalho TS, et al. Non-mammalian models in behavioral neuroscience: consequences for biological psychiatry. Frontiers in behavioral neuroscience. 2015;9:233-33.

35. Mora C, Tittensor DP, Adl S, Simpson AGB, Worm B. How Many Species Are There on Earth and in the Ocean? PLOS Biology. 2011;9(8):e1001127.

36. Moroz LL, Kocot KM, Citarella MR, Dosung S, Norekian TP, Povolotskaya IS, et al. The ctenophore genome and the evolutionary origins of neural systems. Nature. 2014 Jun 5;510(7503):109-14.

37. Moss AG. The physiology of feeding in the ctenophore Pleurobrachia pileus. In: Williams RB, Cornelius PFS, Hughes RG, Robson EA, editors. Coelenterate Biology: Recent Research on Cnidaria and Ctenophora. Dordrecht: Springer Netherlands; 1991. p. 19-25.

38. Moss AG. Primary inhibition: a mechanism for sudden stoppage of metachrony in ctenophores. Hydrobiologia. 2004 2004/11/01;530(1):155-62.

39. Moss AG, Tamm SL. Patterns of electrical activity in comb plates of feeding Pleurobrachia (Ctenophora). Philos Trans R Soc Lond B Biol Sci. 1993 Jan 29;339(1287):1-16.

40. Müller OF. Zoologiæ Danicæ Prodromus, seu Animalium Daniæ et Norvegiæ indigenarum characteres, nomina, et synonyma imprimis popularium. Havniæ [Copenhagen]: Hallageri. 1776:xxxii + 274 pp.

41. Neal KJ. Pleurobrachia pileus Sea gooseberry. Marine Life Information Network: Biology and Sensitivity Key Information Reviews. [online] Plymouth: Marine Biological Association of the United Kingdom.2005.

42. Pang K, Martindale MQ. Mnemiopsis leidyi Spawning 
and Embryo Collection. Cold Spring Harb Protoc2008.

43. Patry L. W, Bubel MK, Hansen C, Knowles T. Diffusion tubes: a method for the mass culture of ctenophores and other pelagic marine invertebrates. bioRxiv. 2019:751099.

44. Pianka HD. Ctenophora. In: Giese AC, Pearse JS, editors. Pianka HD 1974 Ctenophora In: Reproduction of Marine Invertebrates, Vol 1 Giese AC Pearse JS, eds,pp 201-265 Academic Press Inc, New York. New York: Academic Press Inc.; 1974.

45. Pisani D, Pett W, Dohrmann M, Feuda R, Rota-Stabelli $\mathrm{O}$, Philippe H, et al. Genomic data do not support comb jellies as the sister group to all other animals. Proceedings of the National Academy of Sciences. 2015;112(50):15402-07.

46. Presnell Jason S, Vandepas Lauren E, Warren Kaitlyn J, Swalla Billie J, Amemiya Chris T, Browne William E. The Presence of a Functionally Tripartite Through-Gut in Ctenophora Has Implications for Metazoan Character Trait Evolution. Current Biology. 2016 2016/10/24/;26(20):281420.

47. Roskov Y., Ower G., Orrell T., Nicolson D., Bailly N., Kirk P.M., et al. Species 2000 ITIS Catalogue of Life, 25th March 2019. . Digital resource at www.catalogueoflife.org/col.: Species 2000: Naturalis, Leiden, the Netherlands. ISSN 2405-8858.; 2019.

48. Russell JJ, Theriot JA, Sood P, Marshall WF, Landweber LF, Fritz-Laylin L, et al. Non-model model organisms. BMC Biology. 2017 2017/06/29;15(1):55.

49. Ryan JF, Pang K, Schnitzler CE, Nguyen AD, Moreland RT, Simmons DK, et al. The genome of the ctenophore Mnemiopsis leidyi and its implications for cell type evolution. Science. 2013 Dec 13;342(6164):1242592.

50. Schnitzler CE, Pang K, Powers ML, Reitzel AM, Ryan JF, Simmons D, et al. Genomic organization, evolution, and expression of photoprotein and opsin genes in Mnemiopsis leidyi: a new view of ctenophore photocytes. BMC Biology. 2012 2012/12/21;10(1):107.

51. Simion P, Philippe H, Baurain D, Jager M, Richter DJ, Di Franco A, et al. A Large and Consistent Phylogenomic Dataset Supports Sponges as the Sister Group to All Other Animals. Current biology : CB. 2017 2017/04//;27(7):95867.

52. Tamm S, Tamm SL. Extracellular ciliary axonemes associated with the surface of smooth muscle cells of ctenophores. Journal of Cell Science. 1989;94(4):713.

53. Tamm SL. Ctenophora. Oxford; New York: Clarendon Press; 1982.
54. Tamm SL. Cilia and the life of ctenophores. Invertebr Biol. 2014 2014//;133.

55. Tamm SL, Moss AG. Unilateral ciliary reversal and motor responses during prey capture by the ctenophore Pleurobrachia. J Exp Biol. 1985 Jan;114:443-61.

56. Tamm SL, Tamm S. Ciliary reversal without rotation of axonemal structures in ctenophore comb plates. J Cell Biol. 1981 Jun;89(3):495-509.

57. Tamm SL, Terasaki M. Visualization of calcium transients controlling orientation of ciliary beat. J Cell Biol. 1994 Jun;125(5):1127-35.

58. Wang Z, Thiébaut E, Dauvin JC. Spring abundance and distribution of the ctenophore Pleurobrachia pileus in the Seine estuary: advective transport and diel vertical migration. Marine Biology. 1995 1995/12/01;124(2):313-24.

59. Wickham H. ggplot2: Elegant Graphics for Data Analysis. New York: Springer-Verlag; 2016.

60. Wilke CO. cowplot: Streamlined Plot Theme and Plot Annotations for 'ggplot2'. 2019.

61. Yip SY. Parasites of Pleurobrachia pileus Müller, 1776 (Ctenophora), from Galway Bay, western Ireland. Journal of Plankton Research. 1984;6(1):107-21. 